09

\title{
Спекл-интерферометрическая методика исследования пламени
}

\author{
(C) А.Ю. Попов, ${ }^{1}$ А.В. Тюрин, ${ }^{1,}$ Ф В.Г. Ткаченко, ${ }^{1}$ А.Я. Бекшаев, ${ }^{1}$ В.В. Калинчак, ${ }^{2}$ М.Ю. Трофименко ${ }^{2}$ \\ ${ }^{1}$ Научно-исследовательский институт фризики Одесского национального университета им. И.И. Мечникова, \\ 65082 Одесса, Украина \\ ${ }^{2}$ Кафедра теплофизики Одесского национального университета им. И.И. Мечникова, \\ 65082 Одесса, Украина \\ ฯ e-mail: tyurin@onu.edu.ua
}

Поступило в Редакцию 24 февраля 2019 г.

В окончательной редакции 10 октября 2019 г.

Принято к публикации 24 декабря 2019 г.

Описаны методики регистрации и автоматизированной обработки результатов фазомодулированной спеклинтерферометрии оптически плотных (непрозрачных для собственного излучения) пламен, содержащих конденсированную фазу. Применение скоростной однокадровой методики измерений и специальных процедур обработки позволило определить пространственно-временное распределение показателя преломления, на основании которого можно идентифицировать структуру пламени. В качестве примера приведены результаты исследования факела горения стационарной капли медицинского парафина.

Ключевые слова: пламя, спекл-интерферометрия, скоростная методика, распределение температуры.

DOI: $10.21883 /$ JTF.2020.06.49290.1771

\section{Введение}

Для оптимального использования горючих веществ необходимо иметь детальное представление о механизмах их горения в разных условиях. Известно, например, что для газовых горючих при изменении соотношения окислитель-горючее изменяется структура факела $[1,2]$, при этом изменяются полнота сгорания и рабочие температуры, и, как следствие, меняется эффективность использования горючего, поэтому задача оперативного исследования структуры и диагностики пламени представляет несомненный научный и практический интерес.

Для решения этой задачи успешно применяются бесконтактные дистанционные оптические методы диагностики, которые дают высокое пространственное и временное разрешение при определении структуры факела и распределения в нем температур, не внося возмущений в пламя. Их можно разделить на две группы: в первой исследуется собственное излучение пламени (пирометрия), во второй - пламя просвечивается при помощи внешнего зондового источника оптического излучения (спектроскопия, корреляционная спектроскопия, интерферометрия). Каждой из этих групп присущи свои достоинства и недостатки.

Недостатком пирометрических методов является то, что они предполагают постоянство оптических параметров как пламени, так и промежуточной среды [3]. При их использовании невозможно также учесть внутреннюю структуру факела, так как приходится регистрировать интегральную картину излучения пламени. Таким образом, при неоднородной структуре факела (и, в частности, чередовании в факеле холодных и горячих областей) для ее выявления приходится применять электрические или термоэлектрические методы $[1,2]$. Однако они не являются в полной мере невозмущающими, и с их помощью можно получить информацию только из локального объема факела. Эти недостатки в наибольшей степени проявляются при исследовании оптически плотных, т. е. непрозрачных для собственного температурного излучения, пламен [4].

Зондовые методы второй группы лишены данных недостатков, они обеспечивают регистрацию внутренней структуры пламени и пригодны для исследования даже турбулентных пламен [5-10]. В них для получения информации о внутренней структуре пламени используется картина изменения пространственной неоднородности зондирующего пучка света, прошедшего через пламя. При этом наиболее информативными являются голографические интерферометрические методы диагностики зондирующего излучения. В голографических интерферометрических методах измеряется изменение не амплитуды, а фазы проходящего сквозь пламя зондирующего излучения, и поэтому для данных методик измерения требования к прозрачности пламени значительно ослабляются. Затем, так как коэффициент преломления газообразных сред зависит от температуры, изменение фазы зондирующего света дает информацию о температуре внутри пламени. Кроме этого, такое оптическое зондирование имеет много общего с рентгеновским, в результате чего при обработке экспериментальных данных можно применять весь разработанный для томографии математический аппарат (в частности, преобразование Абеля) и строить не только послойные срезы распределения температур, но и его $3 D$-изображения.

Однако данные методы измерения отличаются большой трудоемкостью и необходимостью использовать дорогое и уникальное оборудование, поэтому насущ- 
ной задачей является разработка альтернативных (более удобных в употреблении) методов, что и явилось предметом настоящего исследования.

\section{1. Постановка задачи}

Цель настощей работы заключается в создании методики интерферометрического исследования пламен, которая была бы удобной и доступной в использовании, но при этом не менее информативной, чем голографическая интерферометрия. Для решения данной задачи мы применили метод фазомодуляционной спекл-интерферометрии, известный как ESPI (Electronic Speckle Pattern Interferometry). Данный метод близок к методу цифровой голографии, но проще, и обладает большей универсальностью. Он легко модифицируется применительно к конкретным характеристикам исследуемых объектов $[11,12]$. Важным его преимуществом является то, что можно исследовать объекты, большие по размерам, чем это допускается при использовании цифровой голографии. Максимальный размер объекта лимитируется только длиной когерентности используемого лазера, в реальности размеры могут варьировать от микрона до нескольких метров. Это позволяет исследовать широкий круг пламен, от горения пылевидных топлив до газовых факелов.

\section{2. Методика ESPI-измерений}

\section{1. Стандартный метод ESPI - реализация и недостатки}

Принципиальная оптическая схема установок для исследования объектов методом ESPI представлена на рис. 1. Фактически это интерферометр Маха-Цандера для пучков со спекловой структурой. Длина волны зондирующего источника излучения 1 (лазера) подбирается таким образом, чтобы объект был прозрачным для зондирующего излучения.

В использованном нами варианте ESPI-метода зондирующий, опорный и суммарный пучки имеют спекловую структуру благодаря наличию в схеме диффузного светорассеивателя 4. Характерной особенностью спекл-структуры является то, что в пределах одного спекла фаза излучения постоянна и меняется случайным образом при переходе от одного спекла к другому [8]. Это, в частности, означает быстрое изменение фазы в „темных“ областях между спеклами, вплоть до возникновения фазовых сингулярностей - винтовых дислокаций волнового фронта [13]. Однако эти сингулярности практически не влияют на поведение фазы в ,яркой“ области спекла, которая представляет основной интерес для измерений. Связанные с „границами“ спеклов нерегулярности фазы могли бы сказаться на пространственном разрешении метода, но оно и так ограничено средним размером спеклов.

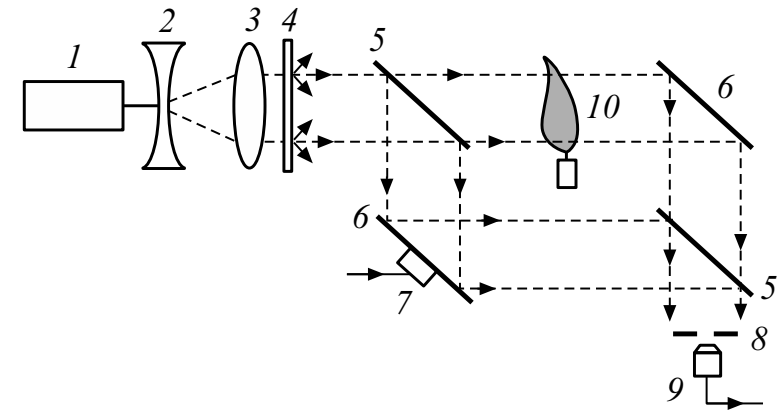

Рис. 1. Принципиальная схема спекл-интерферометра с фазовой модуляцией опорного пучка: 1 - лазер, 2, 3 - линзовые расширители пучка, 4 - диффузный светорассеиватель, 5 - полупрозрачные зеркала, 6 - зеркала, 7 - фазомодуляционное устройство (пьезокерамика), управляемое компьютером, 8 - диафрагма, $9-$ телекамера, $10-$ образец (например, пламя).

При наложении зондирующего и опорного спеклпучков в суммарном пучке (спеклограмме) наблюдаются интерференционные полосы, пространственная частота которых зависит от угла схождения пучков, а расположение узлов и пучностей интерференционных полос от разности фаз пространственно совпадающих спеклов в зондирующем и опорном пучках. При изменении фазы в одном из пучков положение интерференционных полос будет соответственно смещаться. Однако после пространственной фильтрации при помощи диафрагмы 8, когда спекл-поле приобретает развитый характер, интерференционных полос в спеклограмме не наблюдается. В классической интерферометрии это эквивалентно переходу от режима интерференционных полос конечной ширины к полосам бесконечной ширины. В этом случае при изменении фазы опорного пучка (с помощью фазомодуляционного устройства 7) происходит изменение интенсивности (яркости) спеклов в спеклограмме (рис. 2).

Корреляционный анализ изменения интенсивности спеклов таких трех спеклограмм позволяет однозначно определить распределение сдвига фаз в зондирующем пучке относительно фаз в опорном. В дальнейшем такую картину фазовых корреляций зондирующего и опорного пучков будем называть фазовым портретом. Однако поскольку в опорном пучке распределение фаз спеклов не задано, один такой фазовый портрет не дает информации о распределении фазы по фронту зондирующего пучка. Для устранения указанной неопределенности предлагается корреляционный анализ проводить для двух фазовых портретов, которые условно назовем базовым и объектным. Схематически процедура таких измерений представлена на рис. 3 .

Базовый фазовый портрет строится при невозмущенном состоянии зондирующего пучка (пламя отсутствует), а объектный - в присутствии пламени. Корреляционная обработка базового и объектного фазовых 

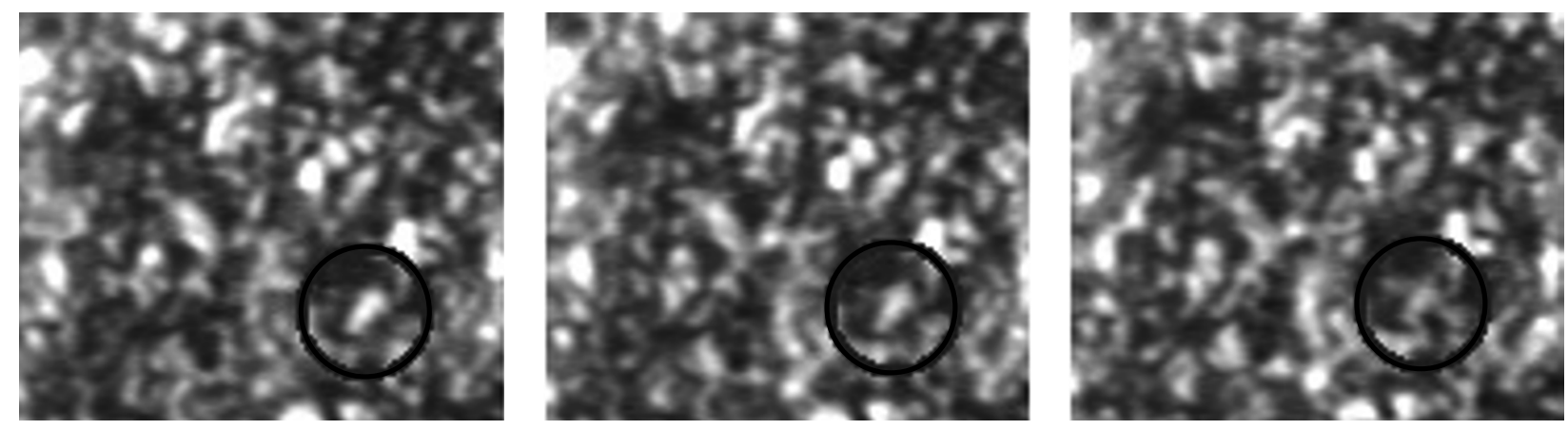

Рис. 2. Набор из трех спеклограмм со сдвигом фазы опорного пучка $0, \pi / 2$ и $\pi$ соответственно. Кружочком выделен спекл, для которого при этом имеет место заметное изменение интенсивности.
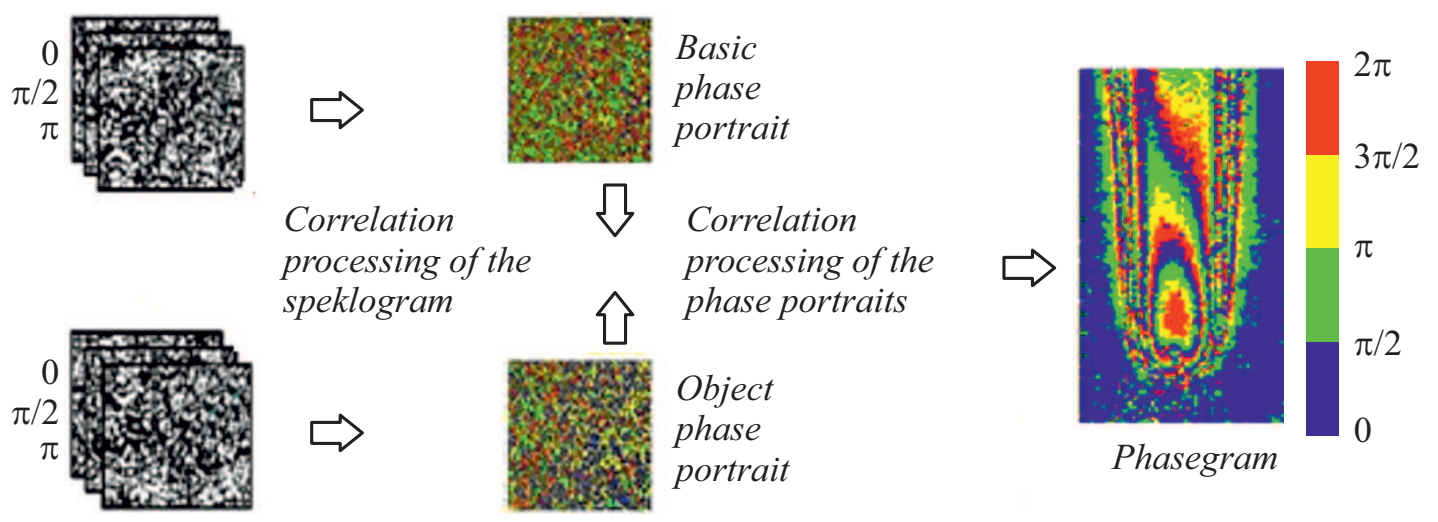

Рис. 3. Схема измерений (пояснения в тексте).

портретов, полученных в последующих циклах измерений для различных моментов времени горения пламени, позволяет однозначно реконструировать распределение фазы по фронту зондирующего пучка (фазограмму), а также фазовые изменения в каждой точке плоскости регистрации для различных моментов времени. На рис. 3 пространственное распределение фазы от 0 до $2 \pi$ в фазограмме, полученной в результате корреляционной обработки фазовых портретов, кодируется цветами, как показано на вертикальной шкале справа: от нуля до $2 \pi$ с инкрементом $\pi / 2$.

Применение этого метода дает очень хорошие результаты при исследовании квазистационарных процессов, но не позволяет исследовать динамические процессы горения. Здесь сказывается его серьезный недостаток - малое быстродействие, поскольку в каждом цикле измерений необходимо зафиксировать как минимум три спеклограммы с изменением фазы опорного пучка на $\pi / 2$ между кадрами. При использовании стандартных телекамер длительность цикла измерений составляет более $0.3 \mathrm{~s}$. На этом временном промежутке фаза зондирующего пучка должна меняться не больше, чем на $\pi / 10$. Для преодоления этого недостатка был разработан скоростной однокадровый метод ESPI [12].

\section{2. Скоростной однокадровый метод ESPI}

Особенностью предлагаемого метода является применение оптической схемы мультиплицирования зондирующего пучка (рис. 4), в результате чего на одном кадре одновременно фиксируется три спеклограммы исследуемого объекта, каждая со своей опорной волной с различными значениями фазы.

Фазовый модулятор 3 в процессе измерений не используется, он необходим только для проведения процедуры стартовой фазовой калибровки с применением обычного метода ESPI, на которой определяется начальная разность фаз мультиплицированной зондирующей и трех опорных волн в каждом спекле каждой из трех спеклограмм на кадре. Таким образом, быстродействие метода увеличивается до быстродействия применяемой телекамеры, для стандартных условий это приблизительно 30 кадров в секунду. В реальности быстродействие еще выше, поскольку время фиксации одного кадра меньше 1/30s. Разумеется, вполне возможно и применение специальных скоростных видеокамер, поскольку процедура измерений заключается, по существу, только в фиксации видеоряда.

К сожалению, в данном случае невозможно применять процедуру обработки, используемой в стандарт- 


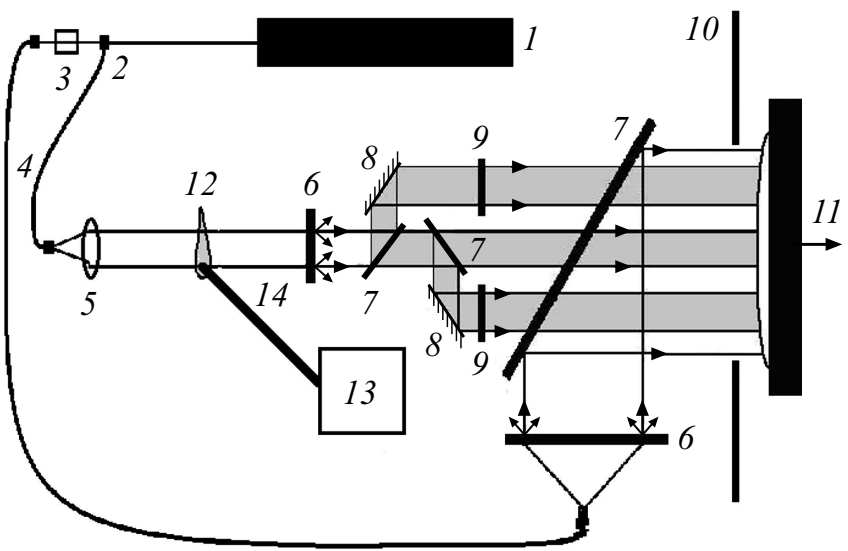

Рис. 4. Схема скоростного однокадрового метода ESPI: 1 - лазер, 2 - светоделитель, 3 - фазовый модулятор, 4 - световоды, 5 - расширительная линза, 6 - диффузный светорассеиватель, 7 - полупрозрачные зеркала, 8 зеркала, 9 - нейтральные светофильтры для выравнивания интенсивностей пучков, 10 - диафрагма, 11 - телекамера, 12 - пламя, 13, 14 - устройства подогрева и подачи горючего (парафина) с капилляром. Серым выделен зондирующий пучок после мультипликации.

ном ESPI-методе, поскольку шаг сдвига фаз для трех опорных пучков не определен, поэтому был разработан специальный алгоритм корреляционной обработки спеклограмм, цель которого состоит в определении фазы $\varphi$ в спекле зондирующего пучка по трем измерениям интенсивности в данном спекле для различных значений фазы опорной волны. Эти значения известны в результате стартовой фазовой калибровки и могут варьировать в пределах от 0 до $2 \pi$.

\section{3. Метод корреляционной обработки спеклограмм}

Запишем световое поле предметной волны в виде

$$
E=A \exp (i \varphi)
$$

где $A-$ амплитуда, а $\varphi-$ локальная фаза для определенного спекла. Опорная волна представляется аналогично

$$
E_{R i}=A_{R} \exp \left(i \varphi_{R i}\right)
$$

Согласно рис. 4, в общем случае существует три опорных волны, которые различаются фиксированными сдвигами фаз $\Delta_{1}$ и $\Delta_{2}: \varphi_{R 0}, \varphi_{R 1}=\varphi_{R 0}+\Delta_{1}, \varphi_{R 2}=\varphi_{R 0}+\Delta_{2}$. Амплитуды опорных волн будем считать одинаковыми; это требование не принципиально (разница в амплитудах легко учитывается при обработке экспериментальных данных), но оно позволяет избежать громоздких выкладок, воспроизведение которых в настоящей работе нецелесообразно.
В спеклограмме интенсивность $I_{i}$ в каждом пикселе будет

$$
\begin{gathered}
I_{i}=\left|E+E_{R i}\right|^{2}=A^{2}+A_{R}^{2}+2 A A_{n} \cos \left(\varphi_{R i}-\varphi\right) \\
=B+D \cos \left(\varphi_{R i}-\varphi\right),
\end{gathered}
$$

где

$$
B=A^{2}+A_{R}^{2}, \quad D=2 A A_{R} .
$$

Функция $I_{i}(x, y)$ описывает распределение интенсивности по спеклам в спеклограмме, полученной при наложении предметной волны и $i$-й опорной волны. Поскольку амплитуды опорных волн во всех случаях одинаковы, величины $B$ и $D$ для всех измерений остаются теми же самыми. Таким образом, в схеме рис. 4 на трех спеклограммах экспериментально наблюдаются распределения интенсивности

$$
\begin{gathered}
I_{0}=B+D \cos \left(\varphi_{R 0}-\varphi\right), \\
I_{1}=B+D \cos \left(\varphi_{R 0}+\Delta_{1}-\varphi\right), \\
I_{2}=B+D \cos \left(\varphi_{R 0}+\Delta_{2}-\varphi\right) .
\end{gathered}
$$

Задача состоит в том, чтобы однозначно определить фазу $\varphi$ в пределах от 0 до $2 \pi$ в каждом пикселе, пользуясь только измеримыми значениями амплитуд $I_{0}$, $I_{1}$ и $I_{2}$.

Принцип алгоритма состоит в следующем. Согласно уравнениям (4) и (5):

$$
I_{10}=I_{1}-I_{0}=2 D \sin \frac{\Delta_{1}}{2} \sin \left(\varphi-\varphi_{R 0}-\frac{\Delta_{1}}{2}\right) .
$$

Отсюда можно определить фазу предметной волны, но неоднозначно:

$$
\varphi=\left\{\begin{array}{l}
\varphi_{1}^{+}=\varphi_{R 0}+\frac{\Delta_{1}}{2}+\arcsin \left(\frac{I_{10}}{2 D \sin \frac{\Delta_{1}}{2}}\right), \\
\varphi_{1}^{-}=\varphi_{R 0}+\frac{\Delta_{1}}{2}+\pi-\arcsin \left(\frac{I_{10}}{2 D \sin \frac{\Delta_{1}}{2}}\right) .
\end{array}\right.
$$

Каждое из чисел $\varphi_{1}^{+}, \varphi_{1}^{-}(8)$ дает решение уравнения (7), т.е. выражает искомую фазу предметной волны через известные или измеримые данные $\varphi_{R 0}, \Delta_{1}, I_{10}$, но только одно из них соответствует истинному значению $\varphi$. Для того чтобы сделать выбор между возможными значениями, достаточно использовать дополнительное уравнение (6). Комбинируя его с (4) и действуя аналогично тому, как было получено (7), находим

$$
I_{20}=I_{2}-I_{0}=2 D \sin \frac{\Delta_{2}}{2} \sin \left(\varphi-\varphi_{R 0}-\frac{\Delta_{2}}{2}\right),
$$

откуда вытекают аналогичные (8) выражения для определения фазы:

$$
\varphi=\left\{\begin{array}{l}
\varphi_{2}^{+}=\varphi_{R 0}+\frac{\Delta_{2}}{2}+\arcsin \left(\frac{I_{20}}{2 D \sin \frac{\Delta_{2}}{2}}\right), \\
\varphi_{2}^{-}=\varphi_{R 0}+\frac{\Delta_{2}}{2}+\pi-\arcsin \left(\frac{I_{20}}{2 D \sin \frac{\Delta_{2}}{2}}\right) .
\end{array}\right.
$$


Здесь тоже только одно из чисел $\varphi_{2}^{+}, \varphi_{2}^{-}$, (в пределах от 0 до $2 \pi$ ) соответствует истинному значению фазы, но теперь для его однозначного выбора существует условие: то же самое значение фазы должно быть и среди чисел $\varphi_{1}^{+}, \varphi_{1}^{-}$. Таким образом, из четырех пар чисел:

$$
\left(\varphi_{2}^{+}, \varphi_{1}^{+}\right) ;\left(\varphi_{2}^{+}, \varphi_{1}^{-}\right) ;\left(\varphi_{2}^{-}, \varphi_{1}^{+}\right) ;\left(\varphi_{2}^{-}, \varphi_{1}^{-}\right)
$$

хотя бы в одной паре числа будут совпадать. Это и будет истинное значение фазы.

На практике величины, входящие в уравнения (8) и (10), определяются путем измерений, и потому содержат случайные (шумовые) составляющие, обусловленные погрешностью измерения. В таких условиях, скорее всего, точного совпадения чисел не получится ни в одной из перечисленных в (11) пар. Тогда наиболее вероятное значение фазы выбирается из той пары, которая содержит два наиболее близкие по значению числа.

Алгоритмически это можно реализовать путем поиска минимума вектора, составленного из всех возможных разностей измеренных значений (8) и (10):

$$
\left[\left(\varphi_{2}^{+}-\varphi_{1}^{+}\right)^{2},\left(\varphi_{2}^{+}-\varphi_{1}^{-}\right)^{2},\left(\varphi_{2}^{-}-\varphi_{1}^{+}\right)^{2},\left(\varphi_{2}^{-}-\varphi_{1}^{-}\right)^{2}\right] .
$$

Предположим, минимальное значение достигается для третьего элемента, т.е. из всех возможных разностей наименьшее абсолютное значение принадлежит величине $\left|\varphi_{2}^{-}-\varphi_{1}^{+}\right|$. В таком случае за истинное значение фазы принимается среднее арифметическое этой пары, т.е.

$$
\varphi=\frac{\varphi_{2}^{-}-\varphi_{1}^{+}}{2} .
$$

Итак, задача сводится к поиску экстремума, поэтому ее можно с самого начала алгоритмизировать как задачу минимизации некоторого функционала. Очевидно, при истинном значении фазы должны одновременно выполняться равенства (7) и (9). Это значит, что выражение

$$
\begin{aligned}
& \left\{\left[I_{1}-I_{0}-2 D \sin \frac{\Delta_{1}}{2} \sin \left(\varphi-\varphi_{R 0}-\frac{\Delta_{1}}{2}\right)\right]^{2}\right. \\
& \left.+\left[I_{2}-I_{0}-2 D \sin \frac{\Delta_{2}}{2} \sin \left(\varphi-\varphi_{R 0}-\frac{\Delta_{2}}{2}\right)\right]^{2}\right\}
\end{aligned}
$$

обращается в нуль тогда и только тогда, когда $\varphi$ равно истинному значению фазы. В условиях реальных измерений с учетом погрешностей истинное значение фазы обеспечивает минимум выражения (14). Его можно найти путем простого перебора всех возможных значений $\varphi$ с некоторым разумным шагом. Еще одно достоинство экстремального алгоритма состоит в том, что можно дополнить (14) произвольным числом аналогичных членов с различными значениями задаваемого сдвига фазы $\Delta_{1}$. Это фактически соответствует проведению большого числа независимых измерений и статистическому усреднению результатов, что, как правило, ведет

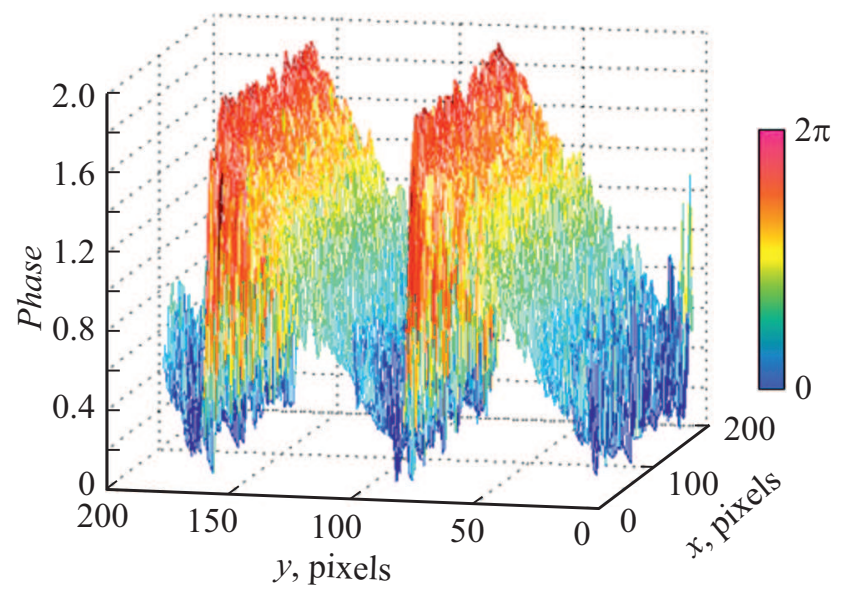

Рис. 5. Распределение фазы модельного объекта, восстановленное по результатам измерений с относительной погрешностью $10 \%$ : по вертикальной оси отложена фаза в единицах $\pi$, цветовая кодировка фазы непрерывна, как показано на шкале справа.

к повышению точности. Если погрешность измерения сигнала в каждом спекле независима, то дополнительное улучшение результатов может быть достигнуто за счет усреднения значений, измеренных в соседних спеклах, что производится с применением различных оконных функций.

Нами разработано программное обеспечение, реализующее данные алгоритмы. В качестве примера на рис. 5 представлены результаты восстановления по разработанным алгоритмам фазы модельного объекта с линейным ростом фазы опорного пучка.

\section{3. Экспериментальное апробирование скоростного метода ESPI}

Для определения возможностей предлагаемого скоростного ESPI-метода он был применен для исследования процесса горения капли парафина. Эта задача достаточно актуальна для изучения механизма горения, к тому же, поскольку данное пламя оптически весьма плотное, она является идеальной для тестирования разработанных методов.

Для создания стационарной капли использовался каркас из тугоплавкой проволоки (хромель) диаметром $0.1 \mathrm{~mm}$, диаметр каркаса составлял $3 \mathrm{~mm}$. Равномерный подвод парафина к каркасу осуществлялся при помощи дозирующего устройства по трубке внутренним диаметром $1 \mathrm{~mm}$.

Получаемые в результате применения ESPI-метода фазограммы подобны интерферограммам (они даже более информативны, в них значение фазы в пределах от 0 до $2 \pi$ определяются однозначно) и могут обрабатываться с применением разработанных для интерферометрии алгоритмов. Однако имеются и существенные различия, 


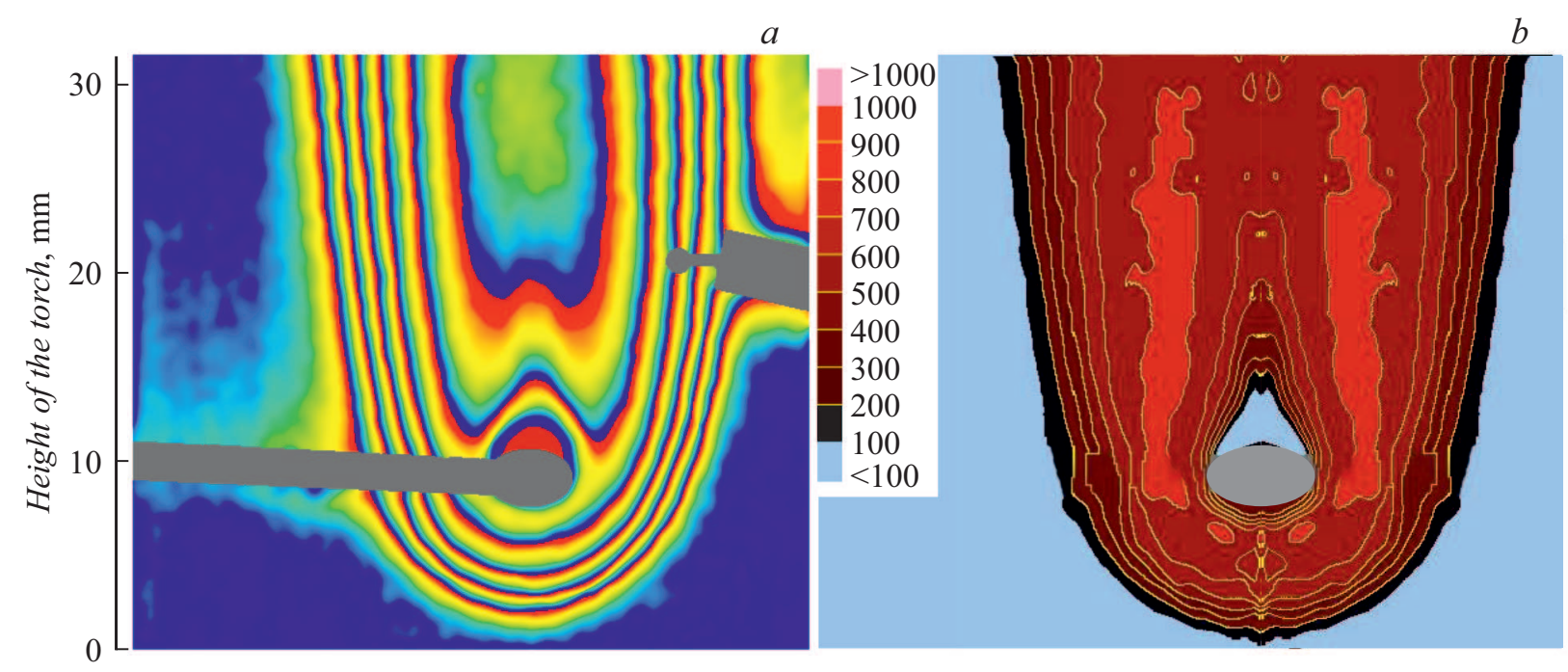

Pис. 6. Фазограмма пламени горения стационарной капли парафина $(a)$ и рассчитанное по ней распределение температур внутри пламени $(b)$ (цветовая шкала показывает температуру в градусах Цельсия). На панели $(a)$ принята та же цветовая кодировка фазы, что и на рис. 5; видны тени подводящей топливо трубки и термопары.

которые необходимо учитывать. При применении спекловых полей интенсивность освещения в некоторых областях спеклограммы падает ниже уровня разрешения (в теории - до нуля), определение фазы в этих областях невозможно (теоретические ограничения). Поэтому при реальном применении данного метода определение фазы становится невозможным в тех областях фазограммы, в которых величина модуляции интенсивности близка к нулю.

Регистрируемые фазограммы фактически являются интегральным отображением пространственного распределения вариаций показателя преломления. Пространственное распределение разности фаз зондирующего пучка до и после возгорания пламени $S(x, y)$ определяется соотношением

$$
S(x, y)=\frac{2 \pi}{\lambda} \int_{Z_{1}(x, y)}^{Z_{2}(x, y)}\left[n_{0}-n(x, y, z)\right] d z
$$

Здесь ось $Z$ совпадает с направлением распространения зондирующего лазерного излучения, $\lambda$ - его длина волны, $Z_{1}(x, y)$ и $Z_{2}(x, y)$ - точки входа и выхода луча из пламени, $n_{0}$ - показатель преломления до возгорания пламени, $n(x, y, z)$ - показатель преломления пламени.

Решив обратную задачу, можно по набору дискретных значений $S(x, y)$ рассчитать пространственное распределение показателя преломления $n(x, y, z)$, на основании которого можно составить карту пространственного распределения неоднородностей в пламени. Если в первом приближении считать пламя химически однородным, то для этого достаточно воспользоваться общей зависимостью показателя преломления газа от температуры и давления, которая выражается формулой

$$
(n-1)=\left(n_{1}-1\right) \frac{P}{760}\left(\frac{1+\gamma P}{1+\alpha t}\right),
$$

где $n$ - показатель преломления при давлении $P$ и температуре $t, n_{1}$ - показатель преломления при нормальных условиях, $P$ - давление [Torr], $\alpha$ и $\gamma-$ коэффициенты, зависящие от газа; для воздуха они составляют $\alpha=3.67 \cdot 10^{-3} ; \gamma=7 \cdot 10^{-7}$.

Детальный расчет распределения неоднородности в объеме пламени требует привлечения методов томографии, что, в свою очередь, требует одномоментного наличия нескольких ракурсов фазовых изображений. В принципе такая задача может быть решена, однако на данном этапе в качестве первого шага мы ограничимся предположением о том, что пламя является осесимметричным, т.е. изменение фазы для всех ракурсов одинаково и описывается равенством (15). Первым следствием этого предположения является симметрия фазограммы, что нередко имеет место в эксперименте (рис. 6). Второе существенное следствие осевой симметрии - это возможность решения обратной задачи для уравнения (15) с помощью преобразования Абеля и его инверсии [9], что значительно упрощает и ускоряет обработку результатов. Кроме того, в обычных условиях вариациями давления в пламени можно пренебречь, и тогда в предположении, что $P$ равно атмосферному давлению, уравнение (16) позволяет однозначно определить распределение самого актуального параметра пламени - его температуры. Именно для таких случаев была проведена серия пробных измерений.

На рис. 6 представлены распределение изменения фазы $S(x, y)$ зондирующего пучка, обусловленное неодно- 
родностью пламени, и соответствующее распределение температур в вертикальной плоскости факела, образованного при горении стационарной капли парафина. На рис. 6 видно, что, несмотря на присутствие значительного количества конденсированных продуктов горения, затрудняющих просвечивание факела, распределение температур получено для всего пламени, кроме совершенно непрозрачных областей капли (серые овалы) и вспомогательных элементов установки: подводящей трубки для подачи горючего и термопары хромель-алюмель, служащей для независимых измерений температуры и калибровки метода. Следующее, что стоит отметить, это высокая степень симметрии фазограммы на рис. 6, $a$, оправдывающая использование алгоритмов томографии на основе преобразования Абеля. Небольшие нарушения симметричной картины пламени связаны с присутствием вспомогательных элементов, однако эти возмущения заметны только для тех ракурсов просвечивания пламени, для которых с точки зрения зондирующего пучка трубка или термопара оказываются сбоку. При усреднении по всем ракурсам эти искажения эффективно подавляются, так что картина распределения температур оказывается гораздо более регулярной. Она показана на рис. $6, b$; заметим, что это фактически „развертка“ полученного в результате обработки фазовых данных радиального распределения, поэтому возможные отклонения от идеальной осевой симметрии пламени на этом изображении не видны.

Рис. 6, a показывает, что даже при „невыгодном“ расположении вспомогательные элементы возмущают главным образом периферию пламени, а распределение температур во внутренних областях их практически „не чувствует“. Это, между прочим, означает, что структура пламени при горении стационарной капли слабо зависит от способа подачи горючего, а выбранный метод измерения температуры внутренних областей с помощью термопары не возмущает температурного поля внутри факела.

Как следует из представленных данных, наиболее горячие области пламени „охватывают“ горящую каплю в виде „чаши“ радиусом примерно $5 \mathrm{~mm}$, высотой около $20 \mathrm{~mm}$ и толщиной „стенок“ 2-3 mm. Видно, что достаточно высокие температуры наблюдаются непосредственно под каплей на расстоянии 2-3 mm от нее; зато сразу над каплей имеет место область низких температур (светло-серое пятно на рис. 6,b). Такая картина согласуется с распределением температур, полученным в [14] при исследовании горения капли метанола методом лазерно- индуцированной флуоресценции.

\section{Заключение}

Судя по результатам тестирования, предложенная методика позволяет определить структуру факела, содержащего большую концентрацию конденсированной фазы, и выявить возмущения, возникающие в факеле как вследствие аэрогидродинамических процессов горения, так и вносимые посторонним механическим вмешательством. Большим преимуществом описанного подхода является его оперативность: в сочетании с быстродействующим ПК он может выполнять десятки измерений в секунду. Это дает возможность не только исследовать динамику быстропротекающих процессов горения, но и целенаправленно контролировать и организовать процесс горения, исходя из поставленных задач. На основе метода возможно развитие разнообразных томографических техник, обеспечивающих наблюдение процессов внутри факела; при этом чувствительность, главным образом к фазе световой волны, делает метод применимым для объектов различной оптический плотности, в том числе сильно поглощающих оптически плотных пламен.

Как показала калибровка температурных измерений с помощью термопары хромель-алюмель, метод обеспечивает относительную погрешность не хуже $5 \%$. По предварительным оценкам, следующим из рис. 6, пространственное разрешение метода не хуже $100 \mu \mathrm{m}$, но дальнейшая оптимизация, несомненно, значительно улучшит этот показатель.

Наибольшие погрешности при определении распределения температур такими методами вносит возможная неоднородность физико-химического состояния и изменение химического состава в различных областях пламени, однако этот недостаток можно преодолеть путем использования при измерениях излучения двух или многих длин волн. С другой стороны, использование нескольких длин волн, хотя и усложняет реализацию метода, способно значительно расширить его возможности, т.к. позволит также измерять пространственное распределение химического и фазового состава, концентрации отдельных компонент и следить за ходом происходящих при горении химических реакций.

Ценное достоинство метода состоит в его способности давать моментальный „снимок“ распределения температуры или других физических параметров сразу по всему объему пламени. Кроме того, он удобно интегрируется в состав измерительных комплексов, использующих другие оптические методы исследования: пирометрические, спектроскопические, люминесцентные и т.д.

\section{Финансирование работы}

Работа выполнена при поддержке Министерства образования и науки Украины, проект 582/18, № государственной регистрации $0118 \mathrm{U} 000198$.

\section{Конфликт интересов}

Авторы заявляют, что у них нет конфликта интересов. 


\section{Список литературы}

[1] Trofimenko M.Y., Aslanov S.K., Smolyar V.P. // Surf. Engineer. App. Electrochem. 2014. Vol. 50. N 3. P. 275-279.

[2] Trofimenko M.Y., Aslanov S.K., Smolyar V.P. // Ukr. J. Phys. 2014. Vol. 59. N 4. P. 359-364.

[3] Свет Я.Д. Оптические методы измерения истинных температур. М.: Наука, 1982. 296 с.

[4] Тробименко М.Ю. Исследование горения твердых смесевых систем оптическими методами // 21-й Междунар. пиротехнический семинар (Москва, Россия, 11-15 сентября 1995). C. $884-890$.

[5] Eckbreth A.C. Laser diagnostics for combustion temperature and species. CRC Press, 1996. $632 \mathrm{p}$.

[6] Thorne A.P. Spectrophysics. London/NY:: Chapman and Hall, 1988. $390 \mathrm{p}$.

[7] Островская Г.В. // ЖТФ. 2008. Т. 78. Вып. 9. С. 1-28.

[8] Франсон М. Оптика спеклов / Пер. с англ. под ред. Ю.И. Островского. М.: Мир, 1980. 171 с.

[9] Флорко А.В., Шевчук В.Г. Спектральные методы исследования высокотемпературных систем. Учебное пособие. Одесса: ОНУ, 2006. 146 с.

[10] Локтионов Е.Ю., Протасов Ю.С., Протасов Ю.Ю., Teлех В.Д., Хазиев P.P. // Инженерный журнал: наука и инновации. 2013. Вып. 10.

URL: http://engjournal.ru/articles/1037/html/index.html

[11] Bulavin L.A., Gotsulskiy V.Ya., Popov A.Y. // Ukr. J. Phys. Opt. 2014. Vol. 15. N 4. P. 216-226.

[12] Пат. Украины на изобретение № 105297. Способ скоростного измерения изменения фазы объектной волны методом фазомодулированной спекл-интерферометрии. Попов А.Ю., Тюрин О.В., Бекшаев О.Я., Гоиульский В.Я. 25.04.2014.

[13] Зельдович Б.Я., Пилипецкий Н.Ф., Шкунов В.В. Обращение волнового фронта. М.: Наука, 1985. 248 с.

[14] Warnatz J., Maas U., Dibble R.W. Combustion: Physical and Chemical Fundamentals, Modeling and Simulation, Experiments, Pollutant Formation. Springer, 2001. 378 c. 